
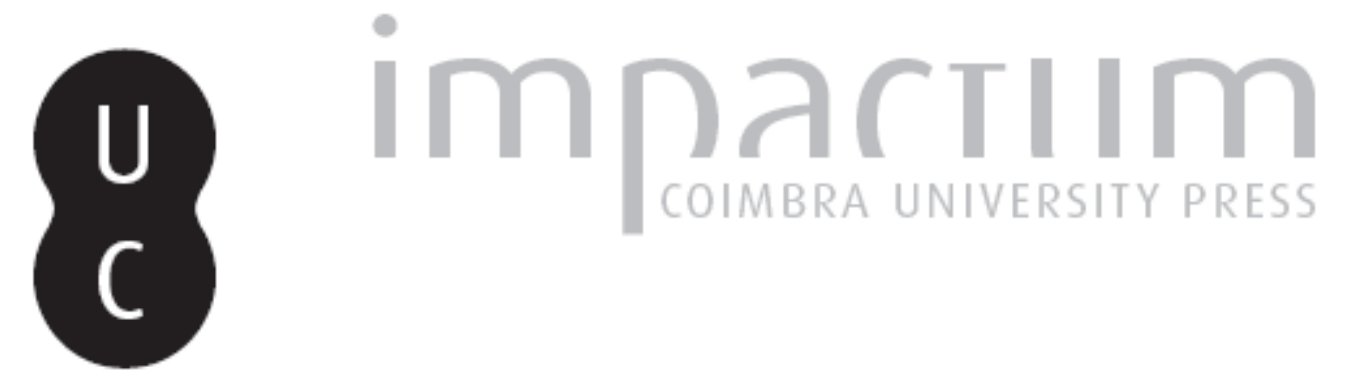

\title{
II nesso tra orexis e facoltà loconotoria nel de anima
}

Autor(es): $\quad$ Palpacelli, Lucia

Publicado por: Annablume Clássica

URL persistente:

URI:http://hdl.handle.net/10316.2/24516

DOI:

DOI:http://dx.doi.org/10.14195/1984-249X_11_5

Accessed : $\quad$ 26-Apr-2023 14:01:04

A navegação consulta e descarregamento dos títulos inseridos nas Bibliotecas Digitais UC Digitalis, UC Pombalina e UC Impactum, pressupõem a aceitação plena e sem reservas dos Termos e Condições de Uso destas Bibliotecas Digitais, disponíveis em https://digitalis.uc.pt/pt-pt/termos.

Conforme exposto nos referidos Termos e Condições de Uso, o descarregamento de títulos de acesso restrito requer uma licença válida de autorização devendo o utilizador aceder ao(s) documento(s) a partir de um endereço de IP da instituição detentora da supramencionada licença.

Ao utilizador é apenas permitido o descarregamento para uso pessoal, pelo que o emprego do(s) título(s) descarregado(s) para outro fim, designadamente comercial, carece de autorização do respetivo autor ou editor da obra.

Na medida em que todas as obras da UC Digitalis se encontram protegidas pelo Código do Direito de Autor e Direitos Conexos e demais legislação aplicável, toda a cópia, parcial ou total, deste documento, nos casos em que é legalmente admitida, deverá conter ou fazer-se acompanhar por este aviso.

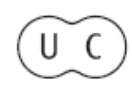



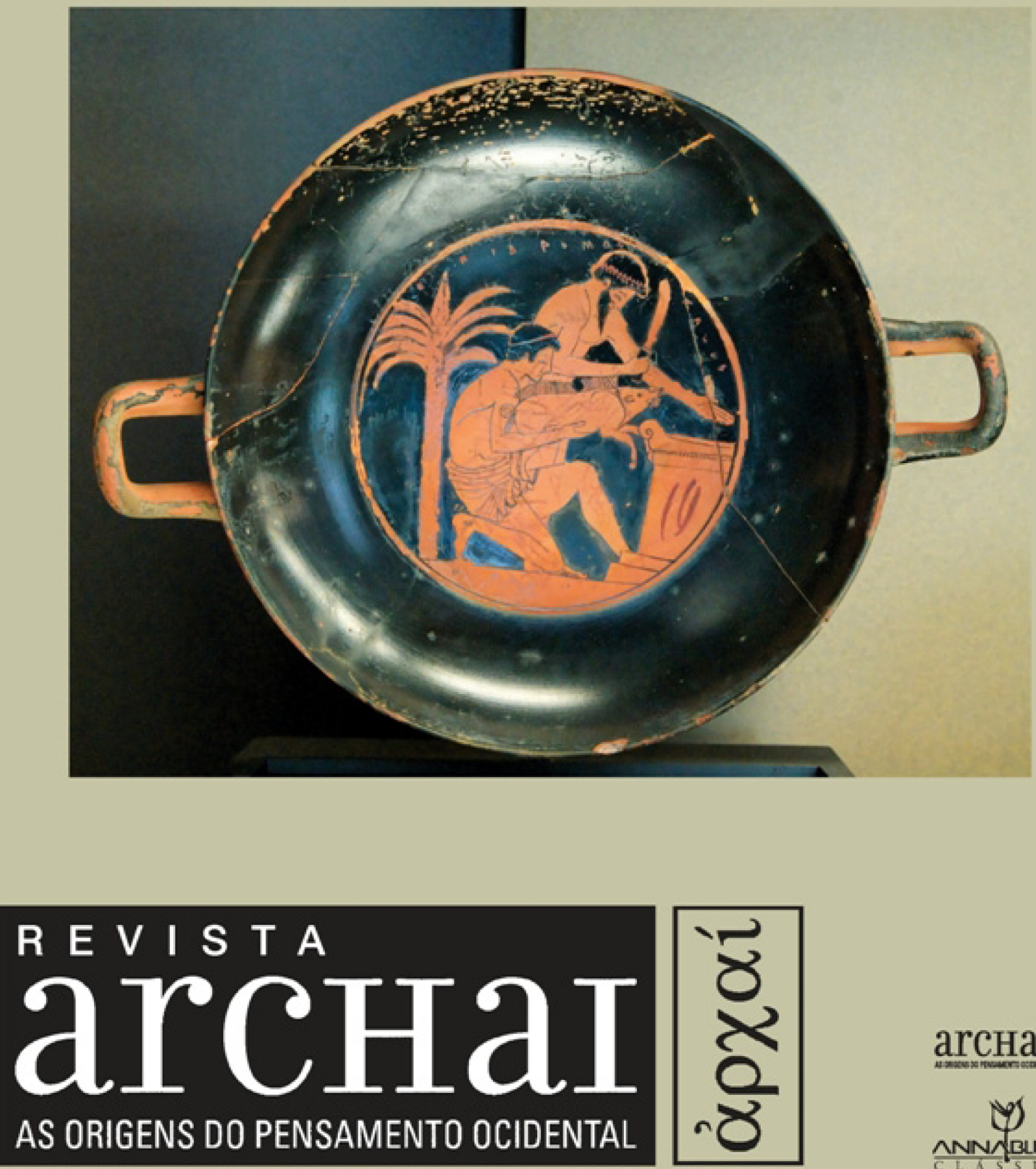

ARCHAI JOURNAL: ON THE ORIGINS OF WESTERN THOUGHT

arcHaI凰

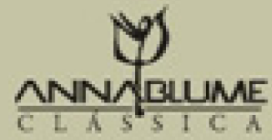




\section{IL NESSO TRA OREXIS E FACOLTÀ LOCOMOTORIA NEL DE ANIMA}

PALPACELLI, L. (2013). Il nesso tra orexis e facoltà locomotorianel De anima. Archai, n. 11, jul-dez, p. 55-62.

ABSTRACT: Questo studio ha per oggetto il nesso tra l'orexis e la facoltà locomotoria che Aristotele stabilisce nel De anima. Egli, attraverso un'argomentazione complessa, indica nella sfera dell'appetizione, la causa della capacità che hanno alcuni animali di muoversi localmente.

L'analisi che propongo si articola, quindi, in due momenti principali:

1. un inquadramento della facoltà locomotoria e di quella appetitiva all'interno della fondamentale tripartizione dell'anima proposta da Aristotele nel De anima (facoltà nutritiva; facoltà sensitiva; facoltà intellettiva);

2. la ricostruzione del ragionamento attraverso cui Aristotele riconduce la dynamis del moto locale principalmente ( $m a$ non unicamente) alla facoltà appetitiva.

PAROLE CHIAVE: Orexis; facoltà locomotoria; phantasia; nous.

ABSTRACT: The subject of this paper is the connection between the orexis and the locomotory skill that Aristotle states in De anima. Through a complex argument, he sees in the appetition the reason why some animals have the skill of moving from a place to another place.

Therefore, my analysis develops in two main points:

3. a presentation of the locomotory and appetitive skill inside the fundamental tripartition of the soul proposed by Aristotle in De anima (nutritive skill; sensitive skill; intellective skill);

4. the reconstruction of the reasoning through which Aristotle connects the dynamis of the local movement mainly (but not only) to the appetitive skill.

KEYWORDS: Orexis; locomotory skill; phantasia; nous.
* Università degli Studi di Macerata.
Lucia Palpacelli *

\section{Una nota introduttiva}

Nell'argomentazione che è oggetto di questo studio risulta fondamentale il concetto di orexis. Credo sia quindi necessario, in via preliminare, delinearlo a grandi linee.

Tale concetto viene definito da Aristotele in modo approfondito nelle Etiche ed egli ripropone un quadro confrontabile a quello delineato in ambito etico, anche nel De anima, pur trovandoci su tutt'altro terreno.

Dato il taglio strettamente fisico-biologico del De anima, mi è sembrato meglio rendere questo termine con la parola "tendenza", che in italiano suona più "neutra" rispetto ad implicazioni etiche, ma condivido le ragioni affermate dalla Fermani per tradurre orexis con "aspirazione" e ne trovo conferma anche nel De anima.

La Fermani sostiene, infatti, che in questo modo «si è cercato di rendere contemporaneamente 1) l'elemento della tensione verso un oggetto e quello della 2) capacità attrattiva esercitata da quell'oggetto stesso» (ARISTOTELE, 2008, p. 1355). Inoltre, così si evita la sovrapposizione con il termine epithumia, cioè il desiderio strictu senso.

Questo problema di traduzione è funzionale ad introdurci alla complessità del concetto di orexis 
che in Etica Eudemia viene così definito: «l'aspira-

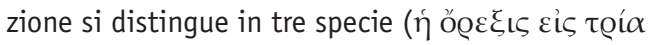

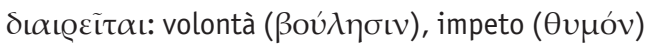

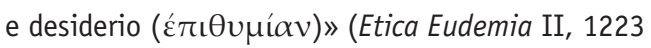
a 26-27). Si può, dunque, pensare all'orexis come ad un genere cui appartengono tre specie ${ }^{1}$.

Come vedremo, il nesso tra l'orexis e la facoltà locomotoria è dato dal fatto che, nel De anima, attraverso un'argomentazione complessa, Aristotele indica nella sfera dell'appetizione, la causa della capacità che hanno alcuni animali di muoversi localmente.

Nell'analisi che segue mi propongo, quindi, (1) un inquadramento della facoltà locomotoria e di quella appetitiva all'interno della fondamentale tripartizione dell'anima proposta da Aristotele nel De anima (facoltà nutritiva; facoltà sensitiva; facoltà intellettiva), (2) per concentrarmi poi sulla ricostruzione del ragionamento attraverso cui Aristotele riconduce la dynamis del moto locale principalmente (ma non unicamente) alla facoltà appetitiva.

\section{Il problema delle parti dell'anima e la difficile collocazione della facoltà locomotoria}

Il problema delle funzioni dell'anima è sentito da Aristotele come uno dei principali riguardo all'anima ed egli lo ripropone costantemente nel corso della trattazione.

Nel libro B del De anima, Aristotele presenta e distingue logicamente due funzioni nell'anima: 1. facoltà nutritiva; 2. facoltà sensitiva, e fa cenno anche ad altre tre dynameis presenti in alcuni esseri dotati dell'anima sensitiva: phantasia, facoltà locomotoria e facoltà appetitiva. Si tratta di funzioni che non possono ricondursi immediatamente alla facoltà sensitiva, anche se il possesso di tale facoltà ne costituisce una precondizione:

Tra gli esseri che hanno la capacità della sensazione,

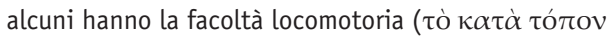

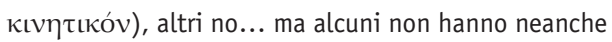
l'immaginazione, altri vivono con questa soltanto (B, 3, 415 7-12).
Analogamente, anche per quanto riguarda la facoltà appetitiva ( afferma in De anima B che la possiedono alcuni tra gli esseri che sono già in possesso della facoltà sensitiva ( $B, 3,414$ b), ma la vede comunque come distinta da questa. Nel libro $\Gamma$ (sezioni 4-5) viene introdotto l'intelletto e alla fine del libro $(\Gamma, 9-10)$ si presenta la facoltà locomotoria.

A questa funzione, dunque, si riserva una sezione a parte alla fine del libro $\Gamma$ e questo è il primo segnale che indica che quella locomotoria è una facoltà problematica per lo Stagirita: essa risulta, infatti, del tutto separata dalle altre facoltà e anche nell'architettonica del De anima sembra posta come qualcosa di "aggiuntivo" ${ }^{2}$.

Con una movenza usuale, Aristotele, nell'incipit della trattazione, propone tutte le problematiche che vanno indagate rispetto a questa dynamis dell'anima:

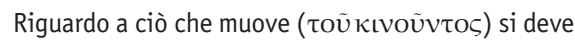
indagare che cosa esso sia dell'anima, cioè $<1>$ se è una sua parte ( $\mu$ ó ııv) separabile o per grandezza o logicamente oppure $<2>$ se è l'intera anima $(\pi \tilde{\alpha} \sigma \alpha \dot{\eta}$ $\psi v \chi \eta ்) ;<3>$ e, se è una certa parte, bisogna ricercare se si tratta di una parte distinta ('́óóvv) da quelle che solitamente vengono nominate e da quelle di cui si è parlato $<4>$ oppure se è una di queste (De anima, $\Gamma$, 9,432 a 18-22).

Alcuni di questi problemi sono gli stessi che nei libri $B$ e $\Gamma$ avevano riguardato le altre funzioni dell'anima (rispetto a tutte le funzioni dell'anima, bisogna capire se siano una parte o se riguardino l'intera anima; se sono separabili, come parti, in senso spaziale o solo logicamente), ma, in questo caso, Aristotele si chiede anche se questa facoltà possa dirsi distinta da quelle di cui ha già parlato oppure se ricada in una di esse. Siamo di fronte a un altro segnale che ci indica la difficoltà nella definizione di questa funzione: lo Stagirita fin da subito si chiede se essa può essere compresa nello schema triadico precedentemente tracciato o se debba esserne distinta.
1. Aristotele, 2008, p. 1233. 2. Hutchinson (1987, p. 374) spiega la posposizione di questa trattazione sostenendo che essa è dovuta al fatto che Aristotele avvicina il percepire e il pensare (dal momento che le considera entrambe forme di cognizione) per cui la sua trattazione assumerebbe il seguente schema: facoltà nutritiva, facoltà sensitiva, pensiero, facoltà locomotoria. A mio parere, è possibile anche un'altra spiegazione che inerisce alla particolare natura della facoltà appetitiva che, come vedremo, viene indicata come causa della facoltà locomotoria. 


\subsection{Una digressione critica: la partizione dell'anima come schema esplicativo}

A conferma che tale facoltà è pensata come "aggiuntiva" rispetto allo schema fondamentalmente triadico di Aristotele, subito di seguito - aprendo quella che a tutti gli effetti è una digressione critica rivolta in particolare verso Platone e i platonici - Aristotele afferma che il problema della parti dell'anima è complesso:

Si ha di fronte subito una difficoltà su come si debba parlare di parti dell'anima e del loro numero. Infatti, da un certo punto di vista, sembra che siano infinite e non solo quelle di cui alcuni parlano distinguendo la parte razionale, passionale e desiderativa; o altri che pongono una parte razionale e una irrazionale.

A sottolineare, di nuovo, l'importanza e la difficoltà del problema posto dalle parti dell'anima, sta anche quest'espressione che indica proprio il dubbio e l'oscillazione che lo Stagirita continua ad avere su questo punto: egli dice infatti «...se si ammettono parti separate dell'anima...». Il tono sembra tornare ad essere quello che si sente già in De anima $\mathrm{A}$, cioè quello di chi pone un problema che lascia aperto. Riguardo a tale problema, potrebbe essere significativo anche il cenno che se ne fa nell'incipit del trattato Sulla giovinezza e la vecchiaia: «Dal momento che altrove si è definita l'anima, è chiaro che l'essenza dell'anima non è come [l'essenza del] corpo, ma ugualmente è evidente che essa è in una qualche parte del corpo e che questa parte è tra quelle che hanno

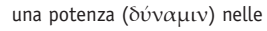
parti [il cuore contiene diverse potenze nelle sue parti, perché è la sede delle diverse dynameis dell'anima], pertanto le altre parti

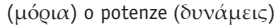
dell'anima, comunque bisogna chiamarle, ora siano tralasciate» (467 b 13-18). Pare riproporsi qui il dubbio di come intendere queste parti o funzioni dell'anima. Piuttosto che di parti dell'anima, osserva Aristotele, sembra meglio parlare di "funzioni", ma qui, il problema si riduce ad essere di natura terminologica. Il dubbio non sembra riguardare tanto la presenza di queste parti o funzioni dell'anima, quanto il modo in cui devono essere chiamate.

Qui lo Stagirita mette a confronto diversi schemi di partizione, ai quali risponde imponendo un suo schema alternativo a quello platonico, che sembra più adeguato, perché individua differenze più nette tra le diverse facoltà dell'anima.

Proprio assumendo lo schema di differenze platonico («secondo le differenze in virtù delle quali essi operano queste distinzioni»), che deriva dall'osservazione delle diverse funzioni dell'anima, lo Stagirita mostra l'inadeguatezza tanto dello schema triadico quanto di quello binario. Egli sostituisce, infatti, alle parti platoniche altre facoltà dell'anima, connotate in senso biologico, e parla di:

1. facoltà nutritiva;

2. facoltà sensitiva;

3. facoltà dell'immaginazione;

4. facoltà appetitiva;

A questo livello dell'argomentazione, meraviglia il fatto che, proprio mentre Aristotele sta trattando della facoltà locomotoria, essa non risulti nell'elenco dato in questo passo. Per ora registriamo questa stranezza per verificare se possa trovare giustificazione in seguito.

Aristotele, in questo passo, denuncia l'inadeguatezza dei modelli di partizione che ha ricordato, ma ci dà anche un segnale dell'inadeguatezza che riguarda la sua fondamentale tripartizione biologica, perché nomina quattro funzioni dell'anima (cui si deve aggiungere l'intelletto che Aristotele qui non considera), delle quali due (immaginazione e facoltà appetitiva) trovano difficile collocazione all'interno dello stesso schema triadico che egli stesso propone nei libri $B-\Gamma$, tanto che Aristotele le elenca come funzioni distinte.

Del resto, lo Stagirita, nel passo appena citato, riconosce che il primo problema che scaturisce per chi ammetta una partizione dell'anima, riguarda la possibilità di porre un numero infinito di parti. Nell'osservare questo egli sembra riproporre il dubbio che è già di Platone nella Repubblica (443 D): anche Platone ipotizza, infatti, che possano esserci parti intermedie dell'anima, rispetto alle tre da lui indicate:

Raccordando le tre parti dell'anima come se fossero tre suoni di un'armonia: l'alto, il basso e il medio e altri ancora intermedi, se mai ce ne fossero, legati insieme tutti questi elementi e diventando interamente uno di molti (443 D 6-E 3).

Questo rende chiara la natura di modello, di schema esplicativo che queste partizioni assumono: si tratta di capire perché un'unica realtà possa 
assolvere funzioni diverse e Platone, nel contesto etico-politico della Repubblica descrive l'anima con uno schema funzionale che prevede tre parti, ma si dice aperto alla possibilità che possano rivelarsene anche altre ${ }^{4}$.

Aristotele dimostra di muoversi allo stesso modo: egli descrive l'anima da un punto di vista fisico-biologico e individua sostanzialmente tre funzioni che, da questo punto di vista, sembrano dare un quadro adeguato di tutte le funzioni dell'anima, ma il fatto che, come si è già accennato, la phantasia e la facoltà locomotoria non possano esservi immediatamente ricondotte suggerisce che non c'è un unico schema possibile ${ }^{5}$. Del resto, tali facoltà cui si aggiunge l'appetitiva - vanno a definire esseri particolari tra $i$ viventi dotati di senso, cioè non sono dynameis che tutti i viventi dotati di senso possiedono. Quindi, per una descrizione generale della realtà bastano le tre facoltà distinte, poi il modello aristotelico si allarga via via adeguandosi alla realtà e va ad individuare altre facoltà che, pur avendo come base la sensitiva, non vi si riducono, ma ne sono distinte.

\section{La problematica natura della facoltà locomotoria}

Dopo la digressione critica appena analizzata lo Stagirita focalizza il discorso sulla facoltà locomotoria: "E rispetto a ciò di cui si interessa il discorso ora, che cos'è che muove l'animale secondo il luogo?" ( $\Gamma, 9,432$ b 8).

Questa dynamis viene definita dopo un vaglio di ipotesi che ripropone lo schema triadico dell'anima con un risultato fallimentare.

A muovere l'animale localmente:

1. non è la facoltà nutritiva: il movimento locale, infatti, è sempre in vista di qualcosa ed è accompagnato dall'immaginazione (che non c'è senza

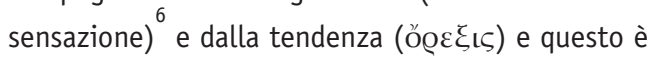
evidente: “Infatti un essere che non desideri o non fugga nulla non si muove, se non di moto violento" $(\Gamma, 9,432$ b 16-17).

In questo contesto in cui Aristotele lavora per esclusione, comincia a darci anche alcuni elementi "in positivo" circa la facoltà locomotoria: essa si accompagna all'immaginazione e all'orexis. Questo fa escludere automaticamente la facoltà nutritiva, perché l'immaginazione e la tendenza, come si è visto, presuppongono che l'essere vivente abbia sensazione.

Inoltre, anche le piante saprebbero, allora, muoversi e avrebbero un organo preposto a questo;

2. non è la facoltà sensitiva, perché ci sono molti animali forniti di sensazione, che, tuttavia, sono stazionari e immobili. Dato che la natura né fa nulla invano né omette nulla di ciò che è necessario, questi animali dovrebbero invece possedere sempre anche parti organiche per la locomozione;

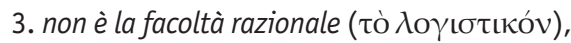

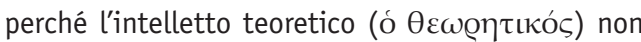
pensa nulla di ciò che è soggetto all'azione e nulla dice su ciò che si deve evitare o perseguire, mentre il movimento è sempre proprio di un essere che evita o persegue qualcosa. Ma neanche quando l'intelletto considera qualcosa di simile, ordina, per questo, di agire (432 b - $433 a)$.

A questo proposito, Aristotele prende in esame due possibilità:

Inoltre, anche quando l'intelletto ( $\tau o \tilde{v} v o \tilde{\text { ) }}$ ordina e la ragione ( $\tau \tilde{\eta} \varsigma \delta\llcorner\alpha v o i ́ \alpha \varsigma)$ dice di fuggire o di perseguire qualcosa, non ci si muove, si agisce, invece,

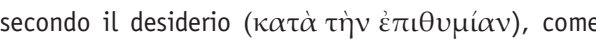
agisce l'incontinente (433 a 2-4).

Questo viene chiarito con un esempio: il medico, pur conoscendo l'arte medica, non sempre la esercita, cioè non basta il fatto di conoscere un'arte per esercitarla. Non è la conoscenza, o non è la conoscenza soltanto, che spinge all'azione. Infatti, Aristotele afferma che non è la scienza ( $\dot{\tau} \tau \iota \sigma \tau \eta \dot{\mu \eta})$ ciò che principalmente (kv@íov) muove ad agire, ma deve esserci appunto un desiderio, una tensione a curare.

Si affronta poi l'orexis in quanto tale: “Ma

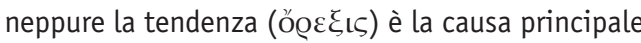

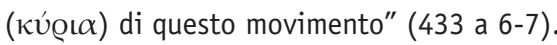

Questo è dimostrato dal fatto che $\mathrm{i}$ continenti, pur essendo soggetti a tendenze e desideri,
4. Su questi temi, cf. Migliori (2007, p. 259), il quale riconosce che «l'invenzione del concetto greco di anima serve a rispondere a due problemi: uno è quello della presenza di una vita che alla fine "se ne va", l'altro è quello dell'unità di un soggetto che ha molte e diverse funzioni psichiche».

5. Per esempio, nell'Etica Nicomachea egli definisce la facoltà nutritiva come la «quarta parte dell'anima» (Etica Nicomachea VI, 12, 1144 a 9). Pe questa segnalazione ringrazio la prof.ssa Arianna Fermani. 6. La phantasia è definita da Movia «una facoltà 'critica' e conoscitiva intermedia tra il senso e l'intelletto» (ARISTOTELE,1979, p. 71), sottolineandone la particolare natura. Infatti, la facoltà sensitiva e la phantasia sono strettamente connesse fra di loro. L'immaginazione

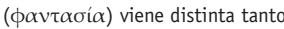
dalla sensazione ( $\alpha \grave{i} \sigma \theta \eta \dot{\sigma \varepsilon} \omega \varsigma$ )

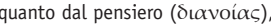
ma non esiste senza la sensazione e, senza l'immaginazione, non può esserci apprensione intellettiva (v́ró $\lambda \eta \psi \iota \varsigma)$, intesa in tutte le sue diverse specie, cioè scienza

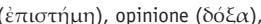

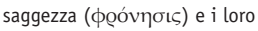
contrari. Quindi, l'immaginazione è diversa, ma è strettamente connessa con entrambi $(\Gamma, 3$, 427 b). 
non fanno ciò verso cui provano una tendenza, ma seguono l'intelletto.

A questo livello, dunque, il problema è stato posto, ma resta irrisolto. Si sono fatte varie ipotesi, tutte scartate, e Aristotele ha poi presentato due

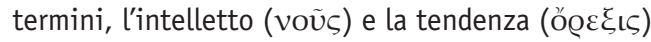
che, in qualche modo, hanno a che fare con il moto locale, ma che, per le ragioni appena viste, non possono esserne indicati come le cause principali.

$\Gamma, 10$ si apre, però, con la constatazione che:

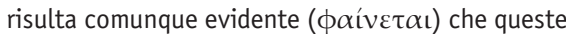
due cose muovono o la tendenza (óp $\varepsilon \xi \xi \varsigma)$ o l'intelletto (voũs), se si può considerare l'immaginazione come una sorta di pensiero (vónoıv) (De anima, $\Gamma, 10$, 433 a $9-10)$.

L'intelletto muove nel caso in cui l'immaginazione si consideri una specie di pensiero. Questo è possibile, perché, spiega Aristotele, molti uomini invece di seguire l'episteme nell'agire, seguono l'immaginazione; gli altri animali poi, non hanno pensiero e non possono ragionare, ma hanno comunque la phantasia che, in questo caso, sembra svolgere per l'azione la funzione che negli uomini svolge

7. A conferma di questo, in De anima $\Gamma, 3$ Aristotele afferma: «per il fatto che [le immagini] restano in noi e sono simili alle sensazioni, molti animali agiscono secondo queste, alcuni perché non hanno intelletto (voũv) come le bestie, altri perché talvolt l'intelletto viene oscurato dalla passione o dalla malattia o dal sonno, come gli uomini» (429

a 4-7). A proposito di questo passo Feola (2012, p. 70) osserva

che «la phantasia è, come la sensazione, un moto: moto ch non avviene senza la sensazione, che cioè avviene solo nei senzienti e solo in quanto senzienti: ha infatti gli stessi oggetti della sensazione».

\section{l'intelletto stesso . Dunque, conclude Aristotele,}

entrambe queste cose muovono secondo il luogo, intelletto e tendenza, l'intelletto che ragiona in vista di

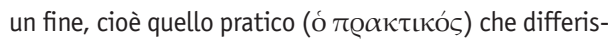
ce da quello teoretico per il fine. Anche ogni tendenza è in vista di qualcosa: infatti la tendenza verso qualcosa

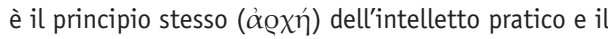

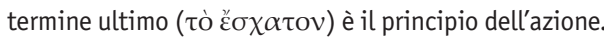
Di conseguenza, è evidente che queste due muovono:

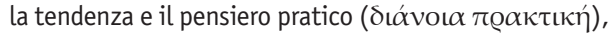

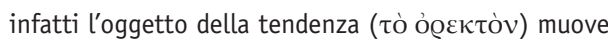

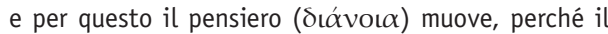
suo principio è l'oggetto della tendenza (De anima, $\Gamma$, $10,433$ a $14-20)$.

Comincia a configurarsi in queste righe una gerarchia di cause motrici che Aristotele ribadisce, chiarendola ed ampliandola, a 433 b 15-20. In quel passaggio egli afferma, infatti, che esistono:

1. motore
2. ciò con cui muove

3. ciò che è mosso.

1. Il motore è duplice: a) motore immobile; b) motore e mosso.

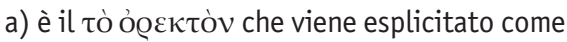
il bene che è oggetto dell'azione;

b) è l'orexis, o come preciserà tra poche righe

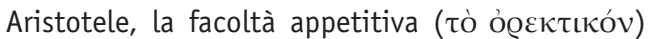
(perché ciò che viene mosso, viene mosso in quan-

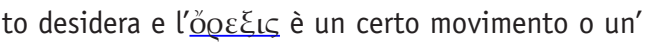
attività realizzata - energheia).

2. ciò con cui la tendenza muove è qualcosa di corporeo, afferma Aristotele, e, stando agli scritti biologici, si ipotizza che si tratti del pneuma innato;

3. ciò che è mosso è l'animale.

In questo schema non trova spazio l'intelletto pratico (Aristotele in $\Gamma, 10$ esclude che quello teoretico potesse essere coinvolto nell'azione) che, dal passo citato, risulta comunque coinvolto (esso viene subito chiamato in causa dall'orexis; del resto Aristotele ha affermato che la facoltà locomotoria è sempre accompagnata da orexis e phantasia che, talvolta, può sostituire l'intelletto) ma ad un livello diverso rispetto alla tendenza: la tendenza verso un dato oggetto muove, infatti, lo stesso intelletto pratico mentre l'oggetto desiderato (quello che Aristotele qui nomina come tò č $\sigma \chi \alpha \tau o v)$ è appunto ciò che, principalmente (come motore immobile), causa l'azione.

Che l'orexis abbia una priorità rispetto all'intelletto, nel processo di movimento locale, lo dimostra anche il fatto che anche l'immaginazione (che, come si è visto, in alcuni casi può "sostituire" l'intelletto), quando muove, non muove senza la tendenza.

Aristotele conclude, quindi, che:

c'è un'unica cosa che muove, la facoltà appetitiva

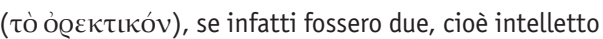

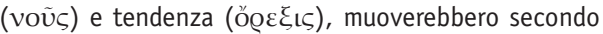
una forma comune (De anima, $\Gamma, 10,433$ a 21-22).

Dunque, se voũc e tendenza fossero entrambe causa del moto locale, si dovrebbe risalire ad una for- 
ma anteriore comune ad entrambi, che li comprenda e non dovrebbe verificarsi questa differenza di livelli di causalità che è stata constata. Essi dovrebbero avere una comune capacità motrice, ma così non è.

a) Infatti, il voũ s non muove senza l'ó@ $\varepsilon_{\xi} \iota \varsigma$, perché, afferma Aristotele, “infatti, la volontà

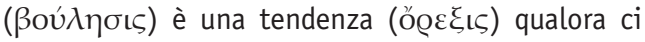
si muova secondo la ragione, ci si muove anche secondo volontà" (De anima, $\Gamma, 10,433$ a 24-25).

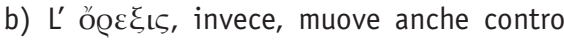
la ragione: ci si muove, infatti, anche secondo il

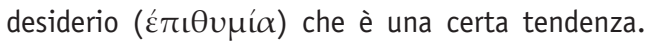

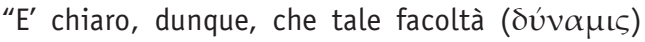
dell'anima muove, la cosiddetta tendenza (ó@ $\xi \xi \varsigma)^{\prime \prime}$ (De anima, $\Gamma, 10,433$ b 1-2).

Aristotele sembra riconoscere, quindi, un primato all'orexis rispetto alla ragione, perché nel movimento e nell'agire, essa sembra avere una potenzialità maggiore rispetto al nous. Infatti, mentre si può agire contro la ragione, mai si può agire contro la orexis, perché anche nella ßoú $\lambda$ $\sigma \iota \varsigma$ - che è la più razionale delle tendenze - si deve, ovviamente, riconoscere la presenza della tendenza. Del resto,

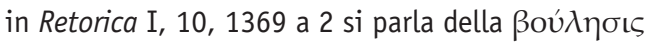
come di un'orexis logistikè.

Qui Aristotele sembra, dunque, precisare meglio quanto aveva affermato in , 9 rispetto al continente: anche in chi agisce secondo la ragione si deve comunque riconoscere la presenza di un elemento che si rifà alla sfera appetitiva.

Dato che, però, può accadere che si generino tendenze contrarie, qualora il ragionamento e il desiderio siano opposti (come accade negli esseri che hanno percezione del tempo e che quindi non riescono a resistere in vista del futuro come ordina l'intelletto, ma si basano sul desiderio presente), il motore deve essere uno per specie ( $\left.\varepsilon^{\prime} \iota^{\prime} \delta \varepsilon\right)$, cioè la

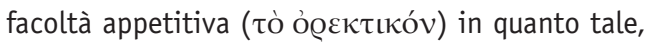
ma molteplice secondo il numero (volontà, impeto, desiderio che, però, come si è visto, si rifanno tutti alla sfera della tendenza).

Si ribadisce, dunque, che la causalità del moto locale va ricondotta, in prima battuta, alla sfera della tendenza non a quella del pensiero. In questo senso, forse, va intesa l'espressione unico per specie.
Come abbiamo già accennato anticipando lo schema di cause motrici stilato da Aristotele, egli, poi, precisa ulteriormente che prima di tutto,

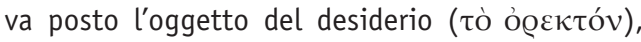
perché muove senza essere mosso, per il fatto che può essere pensato e immaginato. E qui si nota di nuovo quanto orexis e nous o phantasia siano comunque legati a doppio filo, per cui l'una richiama subito gli altri.

Pertanto, in generale, come si è detto, l'animale ha la capacità di tendere e per questo muove se stesso; ma non è capace di tendere senza l'immaginazione. Ma ogni immaginazione è o razionale o sensitiva. Di quest'ultima partecipano, pertanto, anche altri animali (433 b 27-30).

Per spiegare, dunque, questa particolare facoltà dell'uomo e dell'animale Aristotele sembra costruire un modello di cause "a scatole cinesi" che è possibile ricostruire sulla griglia del motore e del mosso che lo Stagirita formula a 433 b:

1. oggetto del desiderio: bene (causa prima del movimento, motore immobile);

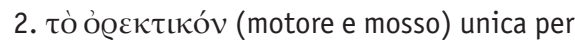

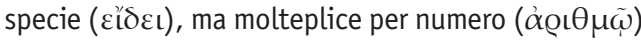

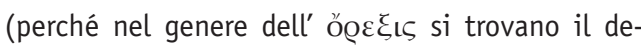
siderio, la volontà e l'impeto). Nell'ambito della facoltà locomotoria, però, tale facoltà, non può essere disgiunta dalla phantasia e dal nous - per $i$ quali costituisce l'archè (cfr. De anima, $\Gamma, 10,433$ a 14-20, citato sopra) - ma, a sua volta, la phantasia e il nous non possono essere disgiunti dall'ó@ع perché l'oggetto del desiderio può essere pensato o immaginato: quindi, tra le due facoltà c'è un rapporto che potremmo definire biunivoco.

3. intelletto pratico che trova il suo principio

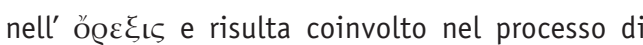
movimento.

4. animale mosso attraverso un mezzo fisico, cioè il pneuma innato.

\section{Considerazioni conclusive}

Il complesso meccanismo che ho appena ricostruito potrebbe spiegare perché la facoltà locomo- 
toria non sia riconducibile unicamente all'orexis come causa principale $(\Gamma, 9)$ : la causa prima è l'oggetto del desiderio, capace di muovere non essendo mossa e di richiamare la facoltà appetitiva, cui Aristotele riconosce una supremazia sulla ragione, come causa locale, ma sottolineando comunque il fatto che l'intelletto e la phantasia sono coinvolti nell'azione e la facoltà appetitiva ne è più o meno condizionata.

Alla luce dell'analisi appena condotta trovano una risposta anche i problemi posti lungo il percorso che ho tracciato: infatti, il fatto che la facoltà locomotoria sia posta, nell'architettonica del De anima, dopo le altre facoltà distinte da Aristotele, può essere spiegato se si pensa che la complessa definizione di tale facoltà attraverso la descrizione della facoltà appetitiva - che fuoriesce dalla fondamentale tripartizione aristotelica, chiamando in causa elementi quali la phantasia stessa e l'intelletto, poteva essere ben compresa solo dopo aver indagato le altre parti.
Infine, il particolare registrato con meraviglia, cioè il fatto che nel passo critico di $\Gamma, 9$, manchi nell'elenco aristotelico proprio la facoltà locomotoria si spiega considerando che, come abbiamo visto, tale facoltà è spiegata da Aristotele ricorrendo alla facoltà appetitiva che, infatti, rientra nell'elenco.

\section{Referências Bibliográficas:}

ARISTOTELE (2008). Le tre Etiche. A cura di A. Fermani. Milano, Bompiani.

ARISTOTELE (1979). L'Anima. Traduzione, introduzione e commento di G. Movia. Napoli, Luigi Loffredo Editore.

FEOLA, G. (2012). Phantasma e Phantasia. Illusione $e$ apparenza sensibile nel De anima di Aristotele. Napoli, Luigi Loffredo Editore.

HUTCHINSON, D.S. (1987). Restoring the order of Aristotle's De Anima, Classical Quarterly, n. 37, p. 373-381.

MIGLIORI, M. (2007). La prova dell'immortalità dell'anima (608 c - 612 C). In: PLATONE (2007). La Repubblica. Traduzione e commento a cura di M. Vegetti. Napoli, Bibliopolis, v. VII, Libro X, p. 199-275.

PLATONE (2007). La Repubblica. Traduzione e commento a cura di M. Vegetti. Napoli, Bibliopolis.

Recebido em setembro de 2012 e aprovado em dezembro de 2013. 